\title{
Pseudoainhum in Systemic Sclerosis
}

MARIANA SILVA, MD, Autoimmunity Department, Serviço de Medicina 2.3, Hospital Santo António dos Capuchos, CHLC, Lisbon; RITA RIBEIRO, MD, Autoimmunity Department, Serviço de Medicina 2.3, Hospital Santo António dos Capuchos, CHLC, Lisbon; JOÃO OLIVEIRA, MD, Autoimmunity Department, Serviço de Medicina 2.3, Hospital Santo António dos Capuchos, CHLC, Lisbon, Portugal. Address correspondence to Dr. M. Silva, Serviço de Medicina 2.3, Hospital Santo António dos Capuchos, Alameda Santo António dos Capuchos, $1169-050$ Lisbon, Portugal. E-mail: mariana.marques.fs@gmail.com. J Rheumatol 2016;43:2076; doi:10.3899/jrheum.160239

Ainhum, also known as dactylolysis spontanea, is an idiopathic development of a fibrotic constricting band in a digit, most commonly affecting the fifth toe. Pseudoainhum refers to the same phenomenon when associated with other conditions, both hereditary and nonhereditary, such as systemic sclerosis $(\mathrm{SSc})^{1,2,3,4}$.

A 54-year-old woman with a previous history of ovarian adenocarcinoma presented with painful edema of the distal half of the fifth left finger. Physical examination revealed 3 digital constricting bands, the strongest located on the second phalange of the fifth left finger. On both hands, cutaneous $\mathrm{SSc}$ of the distal half of the fingers was present (Figure 1 and Figure 2). Over the last 30 years, she had complaints suggesting Raynaud phenomenon (RP) with occasional digital ulcerations. Further investigation revealed mixed connective tissue disease with severe RP. The

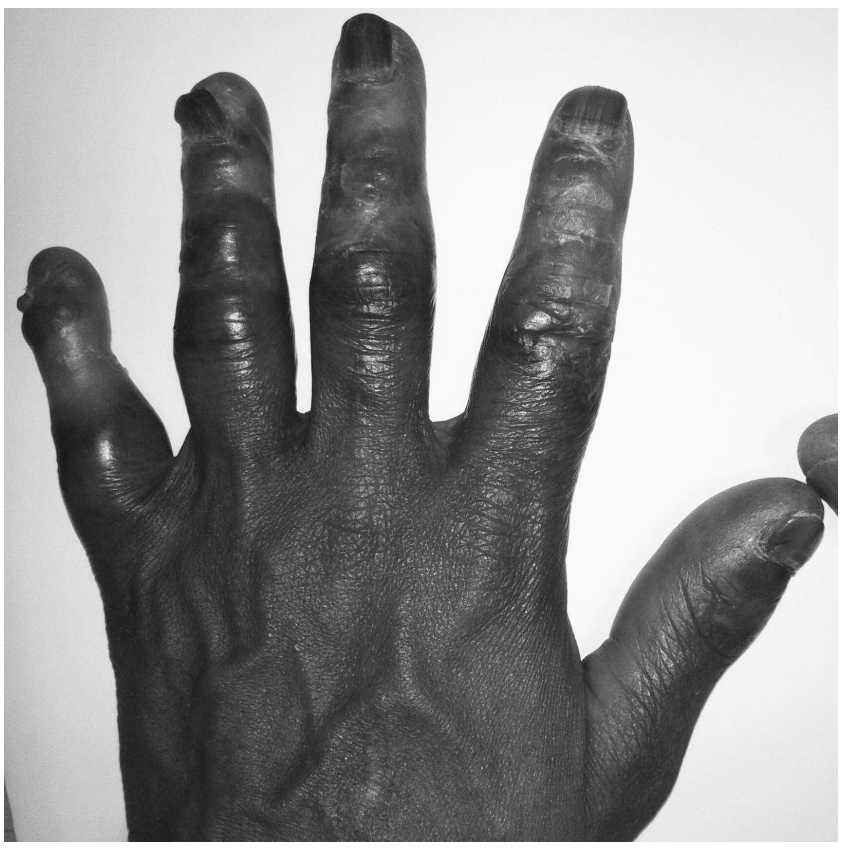

Figure 1. Left hand with cutaneous sclerosis of the distal half of the fingers. above-mentioned annular constrictions were interpreted as pseudoainhum. Iloprost was started, with resolution of the inflammation of the fingers and improvement of RP. Sustained stabilization of the fibrotic constrictive pseudoainhum over a 3-year followup period was observed.

\section{REFERENCES}

1. Rashid RM, Cowan E, Abbasi SA, Brieva J, Alam M. Destructive deformation of the digits with auto-amputation: a review of pseudo-ainhum. J Eur Acad Dermatol Venereol 2007;21:732-7.

2. Park BS, Hyun Cho K, Youn JI, Chung JH. Pseudoainhum associated with linear scleroderma. Arch Dermatol 1996;132:1520-1.

3. Almond SL, Curley RK, Feldberg L. Pseudoainhum in chronic psoriasis. Br J Dermatol 2003;149:1064-6.

4. Kumar P, Gandhi V. Pseudoainhum in psoriasis. Indian J Dermatol 2012;57:238-9.

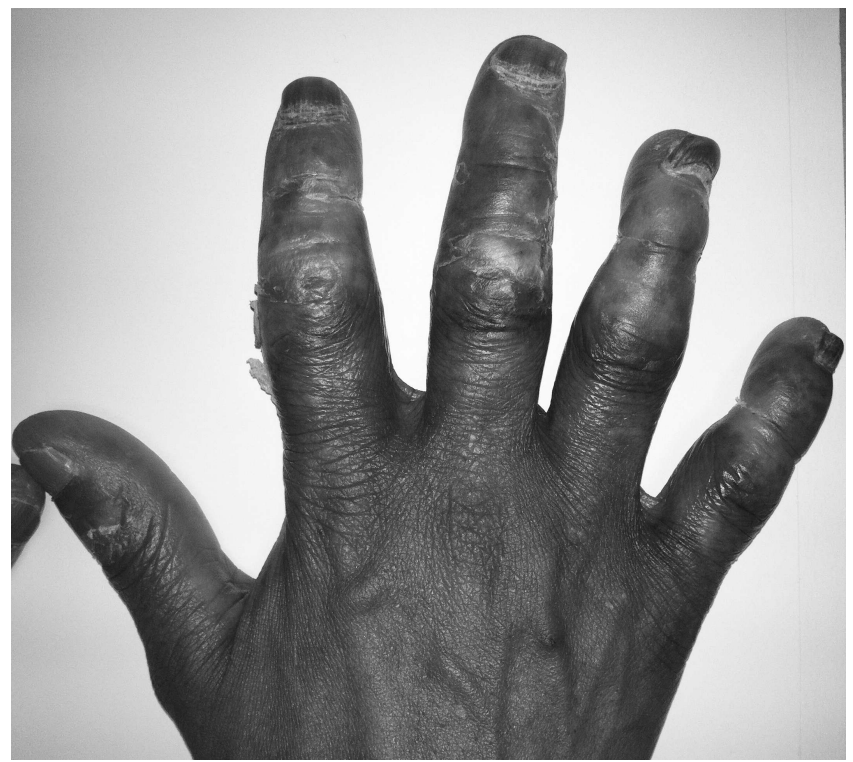

Figure 2. Right hand with cutaneous sclerosis and pseudoainhum of the fifth finger. 